\title{
Justicia juvenil: entre el proteccionismo de Estado y la garantía de derechos
}

\section{Juvenile justice: between State protectionism and the guarantee of rights}

\author{
Hernando HuRTADO RODRÍGUEZ \\ Observatorio del Sistema de Responsabilidad Penal para Adolescentes \\ hhurtadoro@unal.edu.co
}

Recibido: 08/04/2012

Revisado: $13 / 11 / 2012$

Aceptado: 03/12/2012

Disponible on line: 16/04/2013

\section{Resumen}

¿Es la justicia juvenil un problema de visiones jurídicas o de realidades sociales? Este es un interrogante muy importante en la medida que ha guiado el debate actual sobre la justicia juvenil en gran parte de las sociedades occidentales. Los problemas que suscita la justicia juvenil están atravesados por las distintas concepciones jurídicas o sociales que tratan de abordar el tema de los jóvenes infractores de la ley. Por consiguiente, lo que se presenta a continuación es una descripción teórica sobre los dos aspectos fundamentales que han guiado el funcionamiento de la justicia penal juvenil desde mediados del siglo XIX y el transcurso del siglo $\mathrm{XX}^{1}$. Luego en un tercer apartado se presenta algunos aspectos de la realidad social que atraviesa a una gran parte de los adolescentes infractores de la ley en el contexto colombiano para señalar, de esta forma, algunos puntos de discusión y reflexión que pueden ser abordados por una perspectiva holística desde la intervención profesional del Trabajo Social.

Palabras clave: adolescentes infractores, delincuencia juvenil, justicia penal juvenil, Paradigma de la protección integral, paradigma tutelar.

\begin{abstract}
Is juvenile justice a problem of juridical visions or of social realities? This is a very important query to the degree that it has guided the current debate on juvenile justice in a large number of Western societies. The problems that juvenile justice raises are crisscrossed by different juridical or social concepts that seek to address the topic of youthful offenders of the law. Consequently, what is presented here is a theoretical description of the two fundamental aspects that have guided the operation of the juvenile penal justice from the middle of the nineteenth century and throughout the twentieth century. Then, a third section of this article is a presentation of some aspects of the social reality experienced by a large part of adolescent offenders in the Colombian context, as a means of presenting some possible points of discussion and reflection useful for a holistic perspective of professional intervention of Social Work.
\end{abstract}

Keywords: adolescent offenders, juvenile delinquency, juvenile penal justice, paradigm of integral protection, guardian paradigm.

Referencia normalizada: Hurtado Rodríguez, H. (2013): «Justicia juvenil: entre el proteccionismo de Estado y la garantía de derechos». Cuadernos de Trabajo Social, 26(1): 171-180.

Sumario: Introducción. 1. Paradigma o doctrina tutelar. 2. Paradigma o doctrina de protección integral. 3. Paradigma tutelar o paradigma de la protección integral. Un análisis del caso particular de Soacha (Cundinamarca).

4. Referencias bibliográficas.

\section{Introducción}

Este nuevo paradigma requiere de nuevas aperturas mentales y acciones sociales, que establez- can un final y un nuevo comienzo. Es decir, donde se entienda que la ley no funciona como un presupuesto material inmediato que recon-

${ }^{1}$ Este trabajo es el resultado del proyecto de investigación titularo «Entre el paradigma tutelar y el paradigma de protección integral», financiado por la Facultad de Ciencias Humanas (Convocatoria Orlando Fals Borda). 
figura radicalmente la realidad de una sociedad e instituye así una nueva, sino por el contrario se configura como un proceso - que muchas veces es contradictorio- el cual está determinado por el tiempo y el espacio específicos en el que se desenvuelve.

Para abordar el tema sobre la doctrina tutelar y la doctrina de protección integral como marcos conceptuales y procedimentales de una idea más grande, como lo es la justicia penal juvenil, se hace necesario indagar sobre los cambios sustanciales que plantea una doctrina sobre la otra, y hasta qué punto hay una transformación en la visión y en la práctica. Para este propósito se mostrarán los principios que conciben a la doctrina tutelar, y la doctrina de la protección integral, para posteriormente plantear una reflexión sobre las posibles transformaciones paradigmáticas en la administración de la justicia juvenil desde el marco contextual de la Ley 1098 del 2006 promulgada por el gobierno colombiano, y la realidad social que atraviesa la configuración del sujeto adolescente en el país. Esta categoría de adolescentes sujetos de derechos, es fundamental para comprender que la problemática de los adolescentes infractores de la ley es más compleja de lo que pareciera, ya que no se trata solamente de un problema de conducta antisocial de los y las adolescentes; sino al contrario pareciera ser la manifestación sintomática de la entrada en un proceso de crisis existencial de éstos y de la misma sociedad, donde se están produciendo cambios sustanciales en la forma de socializarse de los adolescentes dentro de las sociedades contemporáneas.

\section{Paradigma o doctrina tutelar}

El surgimiento de la doctrina tutelar se puede señalar a mediados del siglo XIX, cuando se presenta como referente jurídico del tratamiento contra los jóvenes delincuentes. Es de señalar, que las nociones sobre cómo se concibe y configura la categoría de joven o niño, han sido ambiguas en la historia occidental, ya que hay que tener en cuenta que la categoría de infancia, como se denomina generalmente en Occidente a los niños, niñas y adolescentes, es una construcción específicamente social, como lo señala Aries Philippe (1987), que por ende ha sufrido diversas configuraciones a través de la historia humana.
El hecho de que la categoría de infancia responda a un complejo entramado social, y a un proceso histórico no lineal, nos sitúa en un momento del siglo XVII, cuando «las sociedades no podían representarse a los niños y niñas, muchísimo menos pensarlos como sujetos sociales» (Barreto, 2009, p. 16). Aquí es imprescindible tener en cuenta que la configuración de la categoría infancia (Philippe, 1987) es fundamental para concebir el contexto y el marco legal sobre el que se va a desenvolver la vida social de la infancia, siendo a partir de ésta como se instituye la categoría de «menor delincuente», que viene a configurar la doctrina de la situación irregular o tutelar. Esta categoría constituye una cosificación y representación social de la infancia, (que va a depender de los distintos momentos históricos por los que transita la vida en comunidad), la cual podrá instituir nuevos espacios y procesos de socialización y relación social, donde la infancia se posicione socialmente.

Es así como el proceso de intervención estatal que cobija a la infancia entre los siglos XIX y XX y que configura el espectro del paradigma tutelar, instaurará un proceder específico sobre la infancia, en términos de una intervención social y estatal sustentada por la incapacidad — socialmente construida- cognitiva y social de los niños y niñas para responder por actos transgresores de la ley. Estas transgresiones son determinadas por el espacio social y simbólico de una determinada sociedad, donde la configuración del ser desvalido y carente de cualquier rasgo racional, se fundamenta en la desigualdad racional que promulgan y exacerban las sociedades modernas del siglo XIX y XX.

Hay un punto conceptual fundamental para poder hablar de la doctrina tutelar, y es el de la distinción entre infancia y menor. Durante el siglo XIX y mediados del siglo XX se generó una dicotomía en las concepciones del niño o niña, (infante) y la de menor; donde la primera se refería a unas condiciones básicas y de desarrollo (familia, salud y escuela) que se distanciaban de la concepción de menor, en la medida que ésta hacía referencia a la no tenencia de estas condiciones básicas. En consecuencia el niño o la niña se hallaban o catalogaban en una situación irregular, que al mismo tiempo les identificaba o rotulaba como un potencial de- 
lincuente que, a su vez, se transformaba en objeto del cuidado, protección y vigilancia por parte del Estado (Barreto, 2009).

En el momento histórico del siglo XIX no hay una distinción clara entre menores delincuentes y adultos delincuentes, que permita configurar un trato diferenciado para ambos; por el contrario, esta indistinción produjo la reclusión de menores y adultos en el mismo lugar, que en consecuencia generó un grave problema de convivencia y de mayor precarización en la situación de abandono que presentaban los menores en ese momento. Las condiciones infrahumanas que estaban viviendo los niños, niñas y jóvenes durante comienzos del siglo XIX permitió el surgimiento de un movimiento reformista en los Estados Unidos, el cual instauraría para el año 1899, el primer tribunal de menores en la Ciudad de Illinois el cual se caracterizaría por permitir cambios sustanciales en el tratamiento del menor delincuente.

Este movimiento reformista fue denominado como los salvadores del niño (Platt, 1982), que desde perspectivas de filantropía, humanitarismo $\mathrm{y}$ de un progreso social gradual, puso en marcha nuevos procesos e instituciones de tratamiento para los denominados menores delincuentes, permitiendo de esta forma «que el sistema de tribunales para menores (...) [representara] un triunfo del liberalismo progresista sobre las fuerzas de la reacción y la ignorancia» (Platt, 1982, p.16). Es así como este movimiento reformista se concibió más allá de un moralismo sin medida, que no transformaba la visión del crimen y la delincuencia en su tiempo, sino «reproducían el mismo modo de ver el crimen y la delincuencia que una generación anterior de reformadores, [solo que] fueron innovadores porque crearon nuevas instituciones y métodos de control social» (Platt, 1982, p. 20). De esta manera, este movimiento es producto de un contexto social, político, económico y cultural específico, como lo es el siglo XIX en Estados Unidos.

Este primer tribunal de menores implicó un cambio sustancial en el modelo de intervención con estos sujetos delincuentes, que permitió generar un nuevo posicionamiento a nivel socio-jurídico, donde los niños, niñas y jóvenes (menores) abandonados y con falta de condiciones mínimas para su existencia, pasaron a ser objeto de intervención social y estatal, que en una buena parte fue instituida en la figura del juez, el cual se convirtió en un nuevo padre, como lo señala Alejandro Bonasso (2002):

El Juez de Menores tenía el papel de «proteger y educar a los desvalidos, encarrilar al descarrilado, proceder siempre, no como un profesor de derecho, ni un erudito en jurisprudencia, sino simplemente como un maestro que enseña, como un abnegado sacerdote que perdona, más aún, como un buen padre de familia (p. 3).

La aparición del tribunal de menores como figura de intervención estatal, configuró una cosificación especial de los niños, niñas y jóvenes (menores) abandonados, y por ende señalados como delincuentes, en la medida que los representó como simples objetos de intervención y modulación, cuyo principal «objetivo formal [...] [era] rehabilitar, reformar y reinsertar en la sociedad aquellos niños abandonados o delincuentes» (De la Iglesia, Velásquez, y Piekarz, 2008 , p. 325). Esta cosificación de la infancia va a ser el principal fundamento social, jurídico y metodológico para institucionalizar una práctica de intervención estatal, marcada por la anomia social de niños, niñas y jóvenes (menores) abandonados, los cuales se prejuzgaban por su condición de abandono y se señalaban como sujetos proclives a transgredir las normas establecidas en el orden jurídico de la sociedad.

Es importante señalar que la categoría de menor, acompañada del adjetivo delincuente, es el principal componente de la doctrina tutelar, en la medida que permitirá configurar una relación asimétrica entre sociedad, Estado e infancia, ya que proclamará la distinción entre incluidos y excluidos del ámbito social; donde «a los incluidos se les llama niños, adolescentes, jóvenes y a los excluidos, menores» (Misle y Pereira, 2003, p. 4). De esta manera, a los menores se les representa como seres incompletos e inadaptados que requieren una ayuda institucional para reincorporarse a la sociedad (Sotomayor, 2005). Es decir, que en la doctrina tutelar se entiende que los niños, niñas y jóvenes son objeto de la protección y tutela del Estado (Salomone, 2009).

\section{Paradigma o doctrina de protección in- tegral}

La doctrina de protección integral puede situarse históricamente a finales del siglo XX, cuando con la promulgación de la Convención 
sobre los Derechos del Niño de 1989, se instaura un proceso de no retorno con una nueva concepción y configuración de la infancia y la adolescencia. Este proceso se había comenzado a gestar desde 1959 con la Declaración de los Derechos del Niño, pero es a partir de la Convención de 1989, cuando se da un giro drástico e indudable, para que los niños, niñas y adolescentes se transformen en sujetos de derechos y al mismo tiempo de deberes. Este proceso tiene un transcurso histórico muy importante que muestra: "finalmente entre las décadas de 1970 y 1980, cobra mayor fuerza la noción del niño como sujeto de derecho» (De la Iglesia et al., 2008, p. 326). Es decir, «esto significa considerar al niño como un sujeto que puede percibir y pensar autónomamente, o sea un sujeto pleno de derecho y no un incapaz representado por adultos» (ídem).

En la conceptualización de la doctrina de protección integral, es fundamental la categoría de infancia y adolescencia, que reconfigura el espacio social y relacional en el que se desenvuelven los niños, niñas y adolescentes dentro de un sistema de justicia juvenil. Este nuevo posicionamiento social y relacional permite dar un vuelco radical, respecto a la anterior doctrina tutelar, frente a la concepción ontológica de la infancia y la adolescencia que se caracteriza principalmente por posicionar al joven como sujeto de derechos y deberes, y donde prima el interés superior de la niñez.

El sustento socio-jurídico que enmarca la nueva concepción ontológica y social de la infancia y la adolescencia está conformado por un gran marco normativo internacional, que comprende:

a) La Declaración de los Derechos del Niño de Ginebra de 1924.

b) La Declaración Universal de los Derechos Humanos de 1948.

c) La Declaración de los Derechos del Niño, proclamada por la Asamblea General de las Naciones Unidas, el 20 de noviembre de 1959.

d) La Convención americana sobre Derechos Humanos o Pacto de San José de Costa Rica.

e) La Convención de las Naciones Unidas sobre Derechos del Niño, 1989.

f) El Convenio relativo a la Protección del niño y a la Cooperación en materia de Adop- ción internacional, pactado en La Haya, en mayo de 1993.

g) El Convenio Interamericano sobre el tráfico internacional de menores.

h) Las reglas mínimas de las Naciones Unidas para la administración de la justicia de menores: reglas de Beijing, resolución 40/33 de 1985.

i) Las directrices de las Naciones Unidas para la prevención de la delincuencia juvenil: directrices de RIAD, resolución 45/113 de diciembre de 1990.

j) La Declaración sobre la mujer y el niño en estados de emergencia y conflicto armado, resolución 3.318/29 de diciembre de 1974 (Observatorio del Sistema de Responsabilidad Penal para Adolescentes, OSRPA, 2010, pp. 53-54).

Este marco normativo internacional ha permitido exigir, reconfigurar y principalmente adaptar los marcos jurídicos nacionales de los diferentes países del mundo que aceptaron constitucionalmente dichas normas. Para el caso de Latinoamérica, se puede encontrar que son diversos los momentos históricos que se dieron para la implementación de estas normas internacionales. Es a partir de la década de 1990, cuando se inicia el proceso de cambio institucional para garantizar y promover los derechos y deberes de los niños, niñas y adolescentes dentro del marco institucional del Estado y la sociedad civil; dando de esta forma,

El carácter constitucional que presentan los derechos del niño en cuanto están asegurados en un tratado internacional de derechos humanos. Tal carácter significa para el Estado la obligación de modificar sustancialmente todas las leyes nacionales que sean incompatibles con las normas de la convención [internacional de los derechos del niño] (Díaz, 2011, p. 166).

Por parte del Estado, la responsabilidad es concreta y tiene que ver con la garantía de los derechos fundamentales de los niños, niñas y adolescentes, cuando a partir de la ratificación de la Convención Internacional de los Derechos del Niño (CIDN):

El Estado asume frente a sus niños y adolescentes la obligación de brindarles protección integral tanto en lo social como en lo jurídico, mediante la adopción de una serie de políticas y acciones tales como legislar en concordancia con los nue- 
vos postulados, y crear las instituciones necesarias en función de su cumplimiento (Castañeda, 2006, p. 4).

La doctrina de la protección integral pretende configurar un nuevo espacio interrelacional, que dé un nuevo estatus a los niños, niñas y adolescentes dentro de la estructura social y simbólica de la sociedad, para que puedan pensarse como sujetos autónomos, libres, con derechos y garantías, y por supuesto, deberes que los posiciona como seres responsables de sus acciones sociales. En esta medida, como competencia del Estado, se debe construir un sistema especial de justicia juvenil que establezca un mínimo de edad para ingresar en el sistema, que para el caso colombiano corresponde a jóvenes entre 14 y 18 años; y donde los menores de 14 años se disponen bajo un sistema de protección especial. Este sistema judicial diferenciado se muestra como un cambio fundamental en la configuración jurídica y social de los niños, niñas y adolescentes, ya que por un lado desdibuja la antigua intervención estatal paternalista de castigo, y por otro lado fundamenta una interrelación del Estado, la sociedad y la adolescencia distinta, donde los jóvenes se presentan como sujetos y no como objetos de la sociedad.

En consecuencia, la nueva doctrina de protección integral es un nuevo referente de acción y pensamiento, en el que la teoría y la práctica se relacionan dependientemente para construir un sistema de justicia juvenil capaz de garantizar el debido proceso judicial y los derechos fundamentales. Esta doctrina enmarca también un nuevo proceder conceptual y existencial sobre los jóvenes que, por diversos motivos o circunstancias, han realizado acciones contrarias a la ley establecida de cada país $\mathrm{y}$ en donde se privilegia la intervención integral del Estado y la sociedad civil, a través de mecanismos de protección social y protección jurídica. Estos mecanismos se refieren, en el primer caso, a las acciones político-administrativas que propician el desarrollo de la libre personalidad, la satisfacción de necesidades básicas, y la garantía de los derechos fundamentales de la infancia y la adolescencia en general sin ningún tipo de discriminación (Misle, et al., 2003). En el segundo caso, se debe garantizar el cumplimiento de lo consagrado en la Convención internacional anteriormente citada, a través de procesos administrativos y judiciales que canalicen las denuncias, den apoyo especializado y permitan el acceso directo de los niños, niñas y adolescentes a información, asistencia y protección para ellos (Misle et al., 2003).

\section{Paradigma tutelar o paradigma de la pro- tección integral. Un análisis del caso parti- cular de Soacha (Cundinamarca)}

Partiendo del postulado fundamental que conlleva la comprensión del cambio sustancial que hay entre el paradigma de la situación irregular y el paradigma de la protección integral, es evidente que no se ha generado como una práctica social e institucional efectiva; es decir, el paradigma de la protección integral no ha pasado de ser un recurso retórico institucional para la administración de justicia juvenil. La imposibilidad de generar prácticas solidas que sustenten el cambio a un nuevo paradigma de tratamiento ante las responsabilidades penales que les competen a los adolescentes, mujeres y varones, puede ser el principal factor constituyente de una situación tan problemática como la delincuencia juvenil.

En el caso particular del municipio de SoachaCundinamarca, con una población de 397.997 habitantes en 2005, con una proyección para 2010 de 455.992 habitantes (Observatorio del Sistema de Responsabilidad Penal para Adolescentes, 2010, p. 14); es decir, que se estima un crecimiento poblacional del 14,5 por ciento durante los cinco años posteriores al dato censal. Del total de la población del año 2005, encontramos que el 49 por ciento corresponde a varones, mientras el 51 por ciento son mujeres. Estos datos estadísticos tomados del Departamento Administrativo Nacional de Estadística (DANE) también muestran que del total de la población para el 2005, 37.100 habitantes están entre los 15 y los 19 años; es decir que aproximadamente un 10 por ciento de los habitantes de Soacha corresponden a adolescentes.

El dato poblacional sobre la cantidad estimada de adolescentes en el municipio de Soacha, se vuelve de suma importancia, en la medida que son estos actores sociales el principal foco de atención. Para tener una comprensión más o menos clara sobre lo que sucede en este municipio con los mismos, es necesario mirar 
algunas cifras porcentuales, para identificar sus principales carencias sociales e institucionales. Como se dijo anteriormente, la población adolescente dentro del municipio es de aproximadamente un 10 por ciento de la población total, que en su mayoría se caracteriza por tener un bajo nivel de escolaridad, siendo que: «es preciso señalar que del total de la población joven (13-26 años) el 54 por ciento no asistía a ningún establecimiento educativo» (OSRPA, 2010, p. 20).

Esta característica es sin duda una de las más relevantes a la hora de revisar el Sistema de Responsabilidad Penal para Adolescentes (SRPA) en Colombia, ya que desde el marco del nuevo código de infancia y adolescencia (Ley 1098 del 2006), se hace un énfasis muy profundo sobre medidas sancionatorias de corte pedagógico y socio-educativo, donde prima el interés del o la adolescente que está en conflicto con la ley. No obstante, al respecto podemos constatar que el municipio de "Soacha cuenta con un total de 184 establecimientos educativos, 20 son estatales y 164 privados, en su mayoría ubicados en sector urbano en franco desequilibrio con el sector rural que tan solo cuenta con tres establecimientos educativos para brindar atención a 1.106 niños(as) y jóvenes entre los 3 y los 17 años» (OSRPA, 2010, p. 20). Por lo tanto, es claro que el municipio no cuenta con los recursos materiales suficientes para satisfacer una demanda social básica, como es el acceso a la educación básica y secundaria de los niños(as) y adolescentes, generando en consecuencia un vacío institucional y social bastante grande.

Fuera de lo anteriormente señalado, se puede observar que la población adolescente posee otro tipo de características sociales que amerita una mayor comprensión, ya que se conjugan tipos de relaciones sociales problemáticas que se encuentran atravesadas por la familia y las redes sociales a las que tiene acceso los adolescentes, mujeres y varones. Algunas de estas características problemáticas están asociadas principalmente con las altas tasas de violencia intrafamiliar; el consumo de sustancia psicoactivas o alucinógenas; la desestructuración familiar; agravado además por el reclutamiento forzado por parte de grupos armados ilegales. Además todo esto atravesado por una característica general de pobreza y falta de oportunidades (OSRPA, 2010).

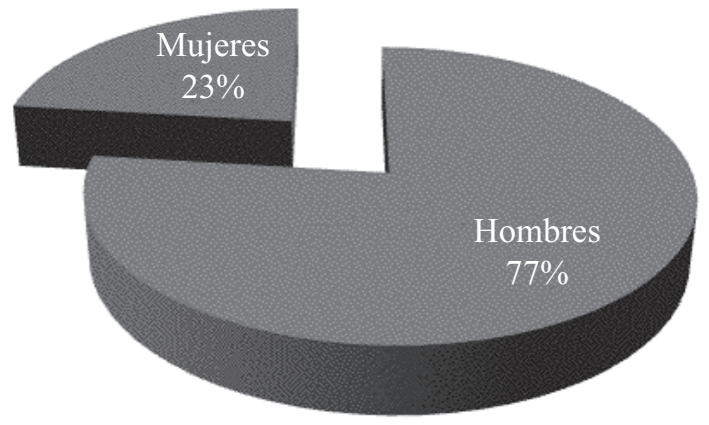

Figura 1.

Fuente: Datos tomados del Observatorio del Sistema de Responsabilidad Penal para Adolescentes. Universidad Nacional de Colombia, elaborado por César Alexis Carrera.

Con relación a la población de adolescentes que se encuentran dentro del Sistema de Responsabilidad Penal para Adolescentes, podemos observar que un 77 por ciento corresponde a varones y un 23 por ciento a mujeres, como lo indica la Figura 1.

Dentro del conjunto de los(as) adolescentes dentro del mencionado Sistema, se puede encontrar que hay un rango de edad de entre los 15 y los 18 años, donde se presentan actuaciones contrarias a la ley por parte de los adolescentes, que se distribuyen en tres problemas concretos. El primero, y más problemático por representar una mayor proporción, tiene que ver con el delito contra la propiedad, más conocido como robo, en el que ubicamos a un 63 por ciento de adolescentes. La segunda gran problemática se refiere a la venta o distribución de sustancias psicoactivas, donde se ubica al 25 por ciento de los adolescentes. Y la tercera problemática está relacionada con los delitos contra la vida y la integridad, en la cual se ubica al 12 por ciento restante de la población juvenil vinculada al Sistema en el municipio de Soacha, como lo indica la Figura 2.

Otro de los aspectos significativos del Sistema en Soacha tiene que ver con la cantidad de ingresos al sistema, ya que lo que podemos encontrar es que un poco menos de la mitad de los adolescentes que están dentro del sistema han ingresado dos o más veces al mismo; lo que permite inferir que no ha sido tan efectivo el proceso pedagógico y socioeducativo que se plantea en el mismo, ya que como se muestra en la Figura 3 una gran parte de los adolescentes que han ingresado en él reingresar. 


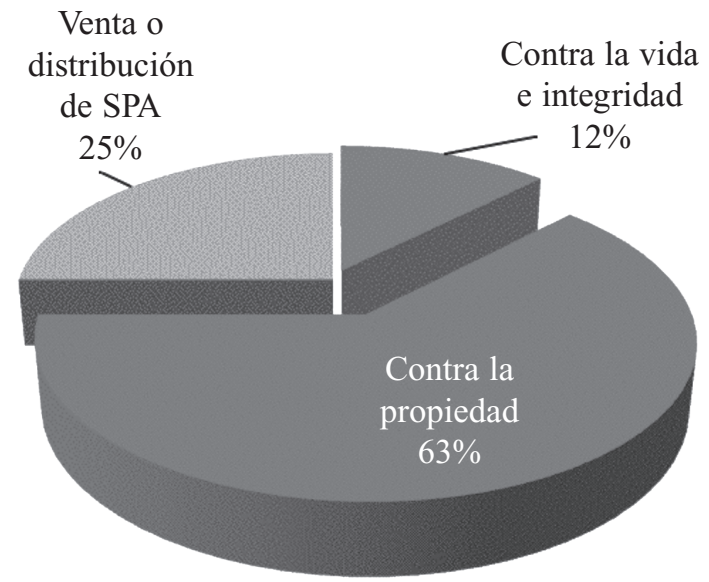

Figura 2.

Fuente: Datos tomados del Observatorio del Sistema de Responsabilidad Penal para Adolescentes. Universidad Nacional de Colombia, elaborado por César Alexis Carrera.

Estos datos de una forma $\mathrm{u}$ otra pueden dar un marco general de la compleja situación que atraviesan los adolescentes infractores de la ley en el municipio de Soacha, que no se aleja del marco general que envuelve la situación general de Colombia frente al tema de la responsabilidad penal juvenil, ya que la constante crítica interna del sistema, y el constante ánimo reformista a la actual ley de infancia y adolescencia (Ley 1098 de 2006), permiten abrir la pregunta sobre qué está pasando con el Sistema de Responsabilidad Penal para Adolescentes y en qué o quiénes recae la responsabilidad de su posible falta de efectividad.

Cabe anotar que el contexto social, político y económico colombiano juega un papel muy importante en la configuración del espacio social y relacional de la sociedad en su conjunto, ya que, teniendo en cuenta problemáticas sociales como el narcotráfico, el conflicto armado interno, la desigualdad social, y el alto grado de pobreza de una gran parte de la población colombiana, solamente son la punta del iceberg de la problemática del país, en la que los niños, niñas y adolescentes también tienen su capítulo. Los problemas asociados al narcotráfico, como el micro-tráfico y consumo de estupefacientes, al igual que otro tipo de problemáticas sociales asociadas al porte ilegal de armas, el secuestro, la extorsión, la violencia de género, la violencia intrafamiliar, la deserción escolar y otras más, configuran el complejo entramado so-

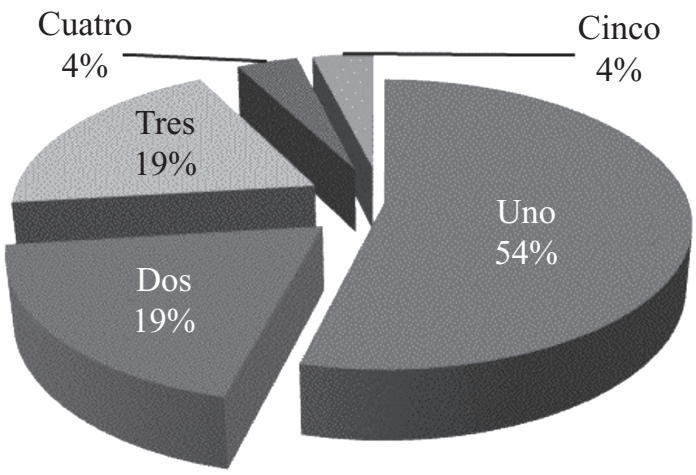

Figura 3.

Fuente: Datos tomados del Observatorio del Sistema de Responsabilidad Penal para Adolescentes. Universidad Nacional de Colombia, elaborado por César Alexis Carrera.

cial en el que se desenvuelven los jóvenes colombianos, donde participan de dos formas: como víctimas o como victimarios.

Es importante señalar que en los diferentes espacios institucionales del Estado - que están directamente relacionados con el Sistema de Responsabilidad Penal para Adolescentes-y la sociedad civil, no es difícil percibir que la categoría de niños, niñas y adolescentes como sujetos de derechos no deja de ser una retórica institucional que se enquista en el discurso oficial de los funcionarios públicos y de las instituciones sociales no gubernamentales que forman parte del Sistema, como operadores para la intervención. Dichas instituciones todavía no han podido generar un cambio en su estructura, que reconfigure un mundo simbólico y significante distinto, que les permita interpretar la complejidad existencial de los adolescentes dentro de un mundo globalizado; pero que al mismo tiempo es segregado por factores culturales, políticos y económicos.

Por lo tanto, es necesario y prioritario en la vía de concebir un nuevo sistema de justicia juvenil, la generación de mejores prácticas institucionales que vayan acordes con el nuevo paradigma de la protección integral; donde se puedan generar relaciones simétricas entre los adolescentes (sujetos de derechos), el Estado y la Sociedad, que permitan un tránsito efectivo hacia nuevas representaciones sociales de los jóvenes, en los que éstos puedan configurar nuevos tipos de identidad - lo cual supone un cambio estructural de la forma en que son 
identificados por el Otro-social-y unas e interrelaciones donde se puedan pensar y autorreferenciar como sujetos de derechos y deberes, provistos de garantías y herramientas efectivas para el libre ejercicio de la ciudadanía.

Teniendo en cuenta la complejidad social sobre la que se sustenta el devenir de la sociedad colombiana - y de tal vez gran parte de las sociedades contemporáneas - no es difícil plantear la existencia de una crisis en el conjunto de la sociedad, que conlleva a transformaciones de tipo social, político y económico, que establecerán nuevas formas de relación y significación dentro del conjunto de actores de una sociedad. Esta presunta crisis se presenta como una lucha de unas fuerzas instituyentes contra otras instituidas, donde la correlación de fuerzas genera diversas tensiones que se interrelacionan en cada espacio de la sociedad. Es así como la problemática de la delincuencia juvenil se puede interpretar como un proceso actual de crisis, donde el paso de una perspectiva socio-jurídica, como lo es el paradigma tutelar, hacia otro paradigma, como es el de la protección integral, está propiciando una «transición del viejo paradigma al innovador, [donde] se desata una lucha por la prevalecía de uno u otro. Ambos discursos de poder, no solo están atravesados por razones [...], sino también por cuestiones políticas, coyunturales y diferentes determinantes institucionales» (De la Iglesia et al., 2008, p. 325).

De esta manera, lo que se puede observar en relación al tratamiento institucional que se realiza a los jóvenes infractores de la ley en el municipio de Soacha-Cundinamarca es que efectivamente no han dejado de percibirse como objetos pasivos de intervención estatal, que requieren ser institucionalizados para poder intervenirles. Un ejemplo de lo anterior podemos evidenciarlo en la Figura 4, donde se observa, que todos los jóvenes que han ingresado en el Sistema de Responsabilidad Penal para Adolescentes han sido remitidos a distintos tipos de instituciones especializadas del mismo; dejando a un lado el principio fundamental del nuevo código de infancia y adolescencia (Ley 1098 de 2006), respecto a la supremacía del interés de los y las adolescentes, y de la primacía de sanciones pedagógicas como el trabajo comunitario; la amonestación; y las reglas de conducta.

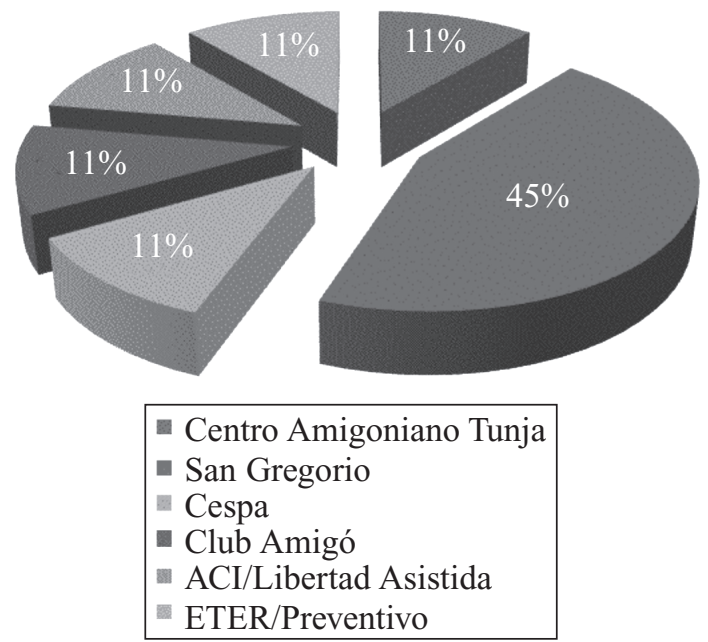

Figura 4.

Fuente: Datos tomados del Observatorio del Sistema de Responsabilidad Penal para Adolescentes. Universidad Nacional de Colombia, elaborado por César Alexis Carrera.

Teniendo en cuenta lo anterior, se puede afirmar, en relación al cambio paradigmático entre lo tutelar y la nueva concepción de protección integral, que hay una plena coexistencia entre ambos paradigmas, en la medida en que se ha institucionalizado un nuevo discurso proteccionista sobre cómo actuar con los jóvenes en conflicto con la ley; pero siguen institucionalizadas prácticas de tipo tutelar, como el paternalismo institucional de remitir a los jóvenes a los centros especializados para su tratamiento, donde además predomina la privación de la libertad como medida sancionatoria privilegiada por los jueces, como se puede evidenciar en el municipio de Soacha. En consecuencia, lo que se ha generado es una postura discursiva:

Nos hemos vestido con los ropajes de este nuevo paradigma, muchas veces perdiendo de vista una mirada crítica sobre las contradicciones existentes entre la práctica y la teoría, entre los universales planteados por la ley y los considerados por los usos y las costumbres, entre las condiciones sociales de producción de subjetividad e identidad y las condiciones particulares de las mismas (De la Iglesia et al., 2008, p. 325).

Es decir, que se ha querido generar un cambio paradigmático en la forma de administrar la justicia juvenil, dejando a un lado la complejidad existencial y social en la que se mueven día a día las personas adolescentes del país. Se 
ha dejado atrás la importancia significante que tiene el medio social, político y económico sobre el que se mueven, que les permiten configurar sus formas de comunicación, relación e interacción dentro del marco de la sociedad.

En consecuencia, es posible plantear que en el proceso de lucha y constante tensión entre uno y otro paradigma ha configurado un paradigma de la transición, caracterizado por la convivencia de prácticas dispares las cuales pueden indicar que:

El paradigma tutelar sigue aún en vigencia, impregnando las concepciones jurídicas, pero también sociales, sobre la niñez. La enunciación institucional y jurídica de los derechos de los niños no es suficiente para contrarrestar la antigua concepción, e instaurar así posibilidades reales para el campo de la subjetividad. La persistencia de los vicios tutelaristas impide el afianzamiento de nuevas representaciones referidas a la niñez, no sólo en las prácticas jurídicas sino también en otras prácticas sociales (Salomone, 2009, p. 2).

Estas prácticas tutelares pueden ser la manifestación de un paradigma en vía de extinción, que en tanto, necesita tiempo y reforzamiento de nuevas prácticas del paradigma instituyente. Por lo tanto, lo que se propone es invitar a pensar y reflexionar sobre: qué acciones concretas podemos empezar a generar, para comenzar a desmontar esas viejas prácticas tutelares, que siguen marcando el devenir de la justicia juvenil en el país; qué hacer para poder avanzar en la implementación efectiva de un paradigma de los derechos, donde haya un vuelco total sobre las formas de relación entre los adolescentes, el Estado y la familia; cómo debemos comenzar a actuar para instaurar un nuevo orden social que apunte al reconocimiento de las diferencias particulares de las personas, mujeres y varones, pero que al mismo tiempo permita que todos y todas los actores de la sociedad puedan acceder y exigir sus derechos en igualdad de condiciones.

De esta manera, para poder generar una mayor aprehensión del nuevo paradigma de protección integral, es necesario dar por sentado que no es un proceso fácil ni a corto plazo, sino por el contrario es un proceso complejo y a largo plazo; en el que se deben integrar diversos factores de tipo social, cultural, político y económico, que son, a la larga, factores determinantes en la composición de las políticas públicas de infancia y adolescencia y de las reformas jurídicas del Estado. Entonces, para que se pueda dar un afianzamiento en la incorporación del nuevo paradigma hay que aceptar que el proceso no comienza ni termina con la promulgación de una nueva ley, ni tampoco pensar que ésta por sí sola cambiará automáticamente las prácticas sociales, culturales y las concepciones mentales de una sociedad y las prácticas burocráticas de las instituciones dónde opera el Sistema de Responsabilidad Penal para Adolescentes; sino que al contrario, se debe partir de la idea de que es un proceso en el que termina un paradigma que ha durado más de medio siglo para instaurar otro nuevo, que requiere tiempo y condiciones materiales y humanas suficientes y capacitadas para su asimilación y puesta en marcha.

\section{Referencias bibliográficas}

Barreto E. (2009). El código de la Infancia y la Adolescencia: elementos para su comprensión e interpretación. En A. Monsalve (ed.), Formación Integral. Ley de Infancia y la Adolescencia: Análisis y perspectivas (pp. 12-37) Bogotá: Universidad Nacional de Colombia.

Bonasso, Alejandro (2002). Adolescentes en conflicto con la ley penal: derechos y responsabilidades (el caso uruguayo) [en línea]. Disponible en: http://www.iin.oea.org/adolescentes_en_ conflicto_con_la_ley_penal_A._Bonasso.pdf.

Castañeda Benavides, Luisa. (2011). El cambio de paradigma en la ley orgánica para la protección del niño y del adolescente. Especial referencia al derecho a la protección en materia de trabajo [en línea]. Disponible en: http://servicio.bc.uc.edu.ve/derecho/revista/idc25/25-7.pdf

De la Iglesia, Matilde; Velásquez, María Eugenia; Piekarz, Walter. (2008). devenir de un cambio: del patronato de menores a la protección integral de los derechos de niños, niñas y adolescentes [en línea]. Disponible en http://www.scielo.org.ar/pdf/anuinv/v15/v15a32.pdf.

Díaz Berríos, Gonzalo. (2011). La ley de responsabilidad penal del adolescente como sistema de justicia: análisis y propuestas [en línea]. Disponible en: http://www.politicacriminal.cl/Vol_06/ n_11/Vol6N11A6.pdf. 
Misle, Óscar y Pereira, Fernando. (2003). Hacia un nuevo paradigma en la educación infantil y juvenil para la prevención y tratamiento del abuso sexual [en línea]. Disponible en: http://www.iin.oea.org/Cursos_a_distancia/explotacion_sexual/Lectura3.NuevoParadigma. pdf.

Platt, M. Anthony. (1982). Los salvadores del niño o la invención de la delincuencia. México: Siglo XXI editores S.A.

Salomone Z. Gabriela. (2009). Infancia y Derechos Humanos en el campo deontológico [en línea]. Disponible en: http://www.seadpsi.com.ar/congresos/cong_marplatense/iv/trabajos/trabajo_167_513.pdf

Observatorio del Sistema de Responsabilidad Penal para Adolescentes (2010) La medida pedagógica como sanción en el sistema de responsabilidad penal para adolescentes (ley 1098 de 2006) estudio socio jurídico. (Proyecto de Investigación, 1). Bogotá: Universidad Nacional de Colombia, Facultad de Derecho, Ciencias Políticas y Sociales, Facultad de Ciencias Humanas. 